\title{
Developing search strategies for clinical practice guidelines in SUMSearch and Google Scholar and assessing their retrieval performance
}

\author{
Andrea Haase*, Markus Follmann, Guido Skipka and Hanna Kirchner
}

Address: Institute for Quality and Efficiency in Health Care (Institut für Qualität und Wirtschaftlichkeit im Gesundheitswesen [IQWiG]), Dillenburger Str. 27, 51105 Cologne, Germany

Email: Andrea Haase* - andrea.u.haase@arcor.de; Markus Follmann - markus.follmann@iqwig.de; Guido Skipka - guido.skipka@iqwig.de; Hanna Kirchner - hanna.kirchner@iqwig.de

* Corresponding author

Published: 30 June 2007

BMC Medical Research Methodology 2007, 7:28 doi:10.1186/147|-2288-7-28

This article is available from: http://www.biomedcentral.com/147I-2288/7/28

(C) 2007 Haase et al; licensee BioMed Central Ltd.

This is an Open Access article distributed under the terms of the Creative Commons Attribution License (http://creativecommons.org/licenses/by/2.0), which permits unrestricted use, distribution, and reproduction in any medium, provided the original work is properly cited.
Accepted: 30 June 2007
Received: I 4 December 2006

\begin{abstract}
Background: Information overload, increasing time constraints, and inappropriate search strategies complicate the detection of clinical practice guidelines (CPGs). The aim of this study was to provide clinicians with recommendations for search strategies to efficiently identify relevant CPGs in SUMSearch and Google Scholar.

Methods: We compared the retrieval efficiency (retrieval performance) of search strategies to identify CPGs in SUMSearch and Google Scholar. For this purpose, a two-term GLAD (GuideLine And Disease) strategy was developed, combining a defined CPG term with a specific disease term (MeSH term). We used three different CPG terms and nine MeSH terms for nine selected diseases to identify the most efficient GLAD strategy for each search engine. The retrievals for the nine diseases were pooled. To compare GLAD strategies, we used a manual review of all retrievals as a reference standard. The CPGs detected had to fulfil predefined criteria, e.g., the inclusion of therapeutic recommendations. Retrieval performance was evaluated by calculating so-called diagnostic parameters (sensitivity, specificity, and "Number Needed to Read" [NNR]) for search strategies.

Results: The search yielded a total of 2830 retrievals; 987 (34.9\%) in Google Scholar and I843 (65.I\%) in SUMSearch. Altogether, we found 119 unique and relevant guidelines for nine diseases (reference standard). Overall, the GLAD strategies showed a better retrieval performance in SUMSearch than in Google Scholar. The performance pattern between search engines was similar: search strategies including the term "guideline" yielded the highest sensitivity (SUMSearch: 81.5\%; Google Scholar: 31.9\%), and search strategies including the term "practice guideline" yielded the highest specificity (SUMSearch: 89.5\%; Google Scholar: 95.7\%), and the lowest NNR (SUMSearch: 7.0; Google Scholar: 9.3).

Conclusion: SUMSearch is a useful tool to swiftly gain an overview of available CPGs. Its retrieval performance is superior to that of Google Scholar, where a search is more time consuming, as substantially more retrievals have to be reviewed to detect one relevant CPG. In both search engines, the CPG term "guideline" should be used to obtain a comprehensive overview of CPGs, and the term "practice guideline" should be used if a less time consuming approach for the detection of CPGs is desired.
\end{abstract}




\section{Background}

An exploding quantity of information, increasing time constraints, and the inadequacy of traditional sources of information underline the importance for clinicians to search efficiently for evidence-based and up-to-date medical information to support diagnostic, prognostic, and therapeutic decision-making processes [1]. Clinical practice guidelines (CPGs) are "systematically developed statements to assist practitioner and patient decisions about appropriate health care for specific clinical circumstances" [2], and are becoming an increasingly familiar part of medical practice [3]. Factors that make searches for CPGs problematic include incomplete indexing in bibliographic databases, as well as the difficulties clinicians encounter in selecting optimal search strategies $[4,5]$. CPG-specific databases exist, such as the NGC (National Guideline Clearinghouse), which includes CPGs that meet defined inclusion criteria and have been published within the previous 5 years [6]. However, so far no search engine is available that searches all guideline databases (e.g. NGC, NHS Clinical Knowledge Summaries [7], Canadian Medical Association Infobase - Clinical Practice Guidelines [8]).

The free-access Internet search engines SUMSearch and Google Scholar are widely used when searching for medical information. SUMSearch was developed by the University of Texas in $1999[9,10]$. It searches the Internet for evidence-based medical information, scanning databases (MEDLINE, DARE, and NGC) as well as various highimpact medical journals [11]. SUMSearch provides validated integrated search filters such as the diagnosis filter developed by Haynes et al [12]. To automate searching, SUMSearch combines meta- and contingency searching. Meta-searching is designed to scan multiple databases and sites simultaneously, and returns one single retrieval document to the user. If too many retrievals are obtained, more restrictive searches (contingency searches) are conducted by activating additional filters. Conversely, if the number of retrievals is too small, SUMSearch adds more databases to the search [13]. The retrievals in SUMSearch are presented in a box with categories arranged from narrative reviews with a broad perspective to publications that are more specific and more difficult to read $[11,14]$. Within the categories, the search results are organised according to database, and are ranked predominantly by publication date in descending order.

Google Scholar is a search engine that was launched in November 2004 by Google Inc. It is available in the beta test-version, which is in continuous transition [15]. Google Scholar is organised according to a so-called federated search [16]: its web crawlers search, process, and index information in the World Wide Web, incorporating it into a single repository, and it refers to this repository to process a search. Google Scholar was developed to provide "a simple way to broadly search for scholarly literature" across many sources (e.g. peer-reviewed papers, books, academic publishers, and universities) [17]. However, further details are not provided; for example, the sources or the search algorithms have not been disclosed, and the term "scholarly" has not been defined [18-22]. In contrast to SUMSearch, Google Scholar presents the search results in a ranked list, the retrievals sorted according to relevance, taking the number of citations into account [17]. However, this system is not strictly applied and may be biased by the high number of citations of older records $[19,23]$. In both Google Scholar and SUMSearch, the search strategy and search results cannot be saved.

Our analysis was motivated by the fact that we had previously not identified studies that compared search strategies for CPGs in SUMSearch and Google Scholar by means of diagnostic parameters. The model for our study was provided by the analysis methods introduced by the Hedges group to detect different types of studies in different databases [12,24-31]. The aim of this study was to provide clinicians with useful search strategies to identify CPGs in SUMSearch and Google Scholar.

\section{Methods}

We compared the retrieval performance (efficient detection of relevant CPGs) of a two-term search strategy in SUMSearch and Google Scholar, using a manual review of retrievals as a reference standard. Our research focussed on nine different diseases currently being evaluated by the German health authorities with regard to their suitability for inclusion in disease management programmes (obesity, osteoporosis, rheumatoid arthritis, Parkinson disease, multiple sclerosis, alcoholism, depressive disorder, schizophrenia, and attention deficit disorder). A "relevant" CPG had to fulfil predefined criteria (e.g. the inclusion of diagnostic or therapeutic recommendations). "Efficient" meant detecting as many unique and relevant CPGs as possible in a given period of time (which varies depending on the time available to the user).

The two primary performance parameters of the evaluation were the completeness of the detected pool of CPGs (measured with the parameter "sensitivity"), and the number of retrievals that had to be read (number needed to read; NNR) to find one relevant CPG. These two measures of retrieval performance represent the (possibly contradictory) situation of a clinician searching for CPGs: i) he or she has sufficient time for a complete review of retrievals to preferably detect all available relevant CPGs on a topic; ii) he or she would like to detect CPGs on a specific condition at short notice while treating a patient. In the former case, the completeness of the search result is 
decisive; in the latter, the time invested is. Specificity (the ability of a strategy to disregard non-relevant retrievals) was defined as a secondary performance parameter.

The individual methodological procedures of the evaluation were continuously developed during the study, according to the experience gained while working with the search engines. These procedures were not specified a priori and were therefore conducted as an iterative process. An overview of the study methodology is shown in Figure 1. We used a two-step approach: (1) the development of a GLAD search strategy (preliminary study); (2) the application of this strategy and the comparison of retrieval performance (main study). The study was conducted in October 2005.

\section{Preliminary study}

Developing the search strategies

First, we entered 14 terms commonly used in guideline searches [32] into SUMSearch and Google Scholar (Table 1 ). The three terms that produced the most retrievals in both search engines were "guideline", "practice guideline" and "standard" (Retrievals SUMSearch, Retrievals Google Scholar in Table 1). We then checked these 14 terms for relevance in combination with the MeSH term "back pain" in SUMSearch (DARE, NGC, and PubMed), using the test term "back pain" as an example of a common disorder and a substitute for the specific disease terms. We restricted this analysis to SUMSearch, since Google Scholar does not solely provide retrievals of medical relevance. We classified a retrieval as "relevant" if the title and abstract of the retrieval included a reference to a CPG on back pain, and the abstract included diagnostic or therapeutic recommendations. Publications identified as meta-analyses or systematic reviews were excluded. The inclusion of a CPG was independent of the methodology of the guideline development (e.g. CPGs based on systematic reviews were not excluded). We recorded the number of retrievals for each term. The terms "guideline", "practice guideline", and "recommendation" produced the highest number of relevant retrievals (CPG term + "back pain" in Table 1), and were therefore defined as the three CPG terms to be used as the first term of the GLAD search strategy. The second term was one of the nine disease-specific MeSH terms mentioned earlier.

\section{Main study}

\section{Application of the search strategies}

The developers of SUMSearch assessed predictors of successful searches for medical evidence and showed that searches were twice as likely to succeed when no more than two terms were included or MeSH terms were used [11]. On the basis of their specifications for an effective search in a search engine, we developed a two-term search strategy combining one of three different CPG terms and one of nine MeSH terms for a specific disease. This resulted in 27 search combinations for SUMSearch and Google Scholar. The components of the search strategy CPG term and MeSH term - prompted us to call this approach the GLAD (GuideLine And Disease) strategy.

We adapted the syntax of the GLAD strategy to the individual requirements of each search engine (Figure 1). In SUMSearch, the truncated CPG terms were combined with the Boolean operator "AND" (1. guideline*, 2. recommendation*, 3. practice guideline*; AND MeSH term). The search was restricted to the category "Practice Guidelines" (NGC and PubMed). Since the PubMed practice guidelines section of SUMSearch does not provide citations, we used the link provided by SUMSearch to search for guidelines at PubMed. DARE had initially been included in the preliminary study, but as no guidelines were retrieved (only systematic reviews and meta-analyses), it was subsequently excluded from the main study.

In Google Scholar, the single or plural CPG terms were used without an operator (1. guideline/s; 2 . recommendation/s; 3. practice guideline/s; MeSH term). We used the advanced interface and selected "in the title". We entered the combination of each CPG term (single or plural) and each MeSH term into the search box "with all of the words". We used the "all in title" restriction, as prior informal searching in Google Scholar had produced an excessive amount of retrievals without this limit.

The retrievals for the nine diseases were pooled. The application of the GLAD search strategy therefore resulted in three retrieval pools (one for each CPG term applied) per search engine.

\section{Defining the reference standard}

After collecting the raw retrievals in SUMSearch and Google Scholar, we defined a reference standard by manually reviewing all retrievals (links) from the retrieval list of each search engine. Each raw retrieval was regarded as a reading unit that had to be reviewed to identify unique and relevant CPGs. Since the GLAD strategies were to be tested against a reference standard, the retrievals had to be unique (i.e., excluding duplicates).

The unique and relevant CPGs of both search engines formed the reference against which each of the three CPGterm search strategies applied in SUMSearch and Google Scholar was tested. As duplicates also involved a relevant input of work and time for the reviewer (each one had to be assessed individually), they were also considered in the analysis (Table 2).

The full-text articles of the retrievals were screened and assessed; if these were not available, abstracts were 


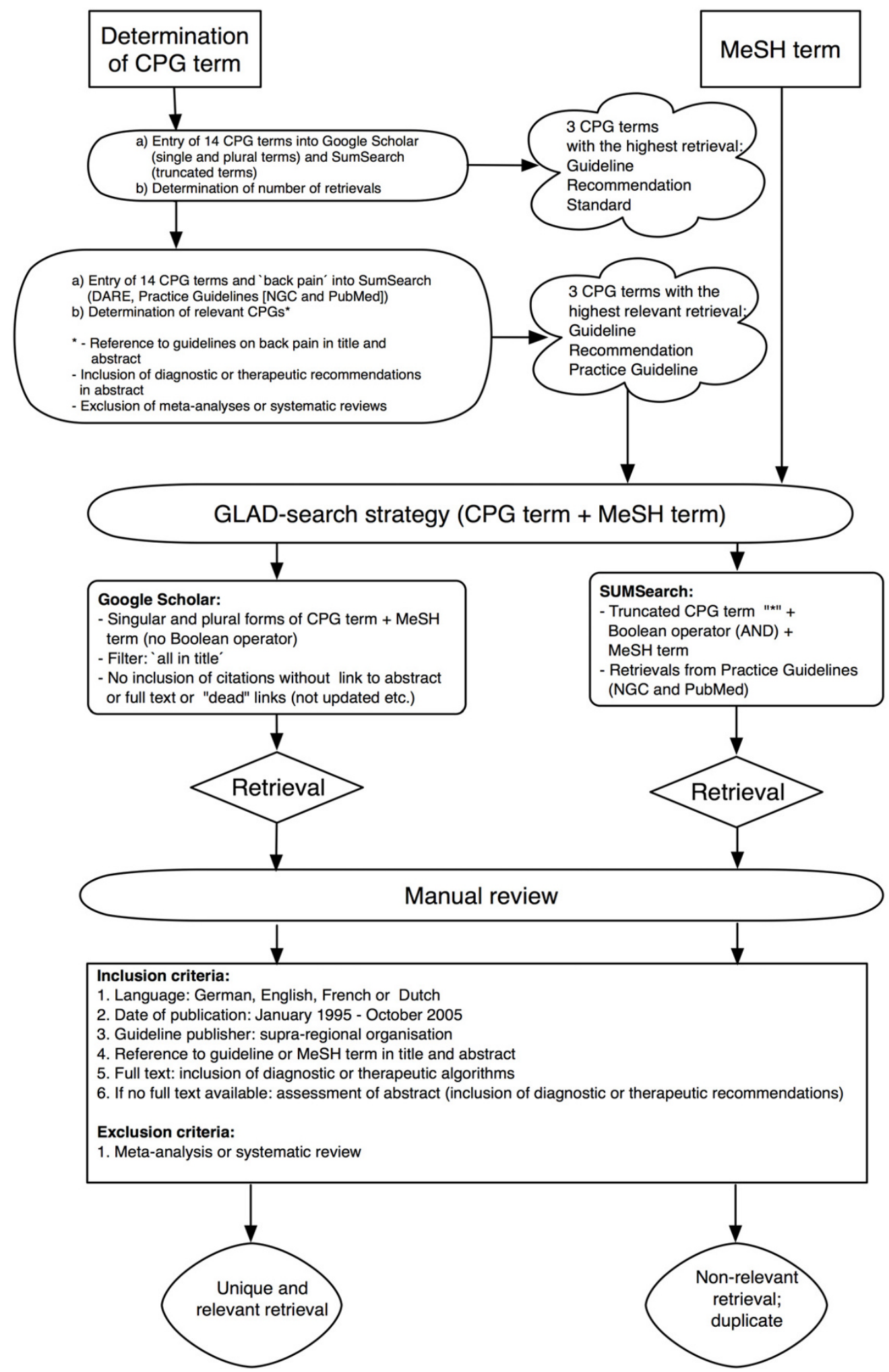

Figure I

Flowchart of study methodology. 
Table I: Determination of clinical practice guideline terms for the GLAD search strategy in the preliminary study

\begin{tabular}{|c|c|c|c|}
\hline $\begin{array}{l}\text { I4 commonly used search } \\
\text { terms }{ }^{\dagger}\end{array}$ & Retrievals SUMSearch $\ddagger$ & $\begin{array}{l}\text { Retrievals Google Scholar } \ddagger \\
\text { (estimated number of retrievals) }\end{array}$ & $\begin{array}{l}\text { Relevant retrievals CPG term } \\
\text { + "back pain"ll (guidelines in the } \\
\text { top } 20 \text { [DARE 24] retrievals) }\end{array}$ \\
\hline Guideline/-s/-* & 340,105 & $1,954,000$ & 58 \\
\hline Practice guideline/-s/-* & 139,385 & 984,000 & 46 \\
\hline Recommendation/-s/-* & 162,239 & $1,892,000$ & 35 \\
\hline Standard/-s/-* & $1,384,650$ & $18,560,000$ & 30 \\
\hline Clinical pathway & 3,332 & 420,000 & 0 \\
\hline Clinical protocol & 76,530 & 572,000 & 0 \\
\hline Clinical standard & 64,009 & 872,000 & 0 \\
\hline Clinical recommendation & 3,649 & 64,900 & 5 \\
\hline Consensus & 62,113 & 970,000 & 7 \\
\hline Clinical consensus & $|2,40|$ & 263,000 & 0 \\
\hline $\begin{array}{l}\text { Consensus (SUMSearch: AND) } \\
\text { development conferences }\end{array}$ & 6,189 & 49,700 & 6 \\
\hline Position paper & 7,115 & $1,740,000$ & 0 \\
\hline $\begin{array}{l}\text { Clinical (SUMSearch: AND) } \\
\text { position paper }\end{array}$ & 1,085 & 272,000 & 0 \\
\hline $\begin{array}{l}\text { Good (SUMSearch: AND) clinical } \\
\text { practice }\end{array}$ & 6,487 & 768,000 & 0 \\
\hline
\end{tabular}

† The terms 'guideline', 'practice guideline', 'recommendation' and 'standard' were entered into SUMSearch and Google Scholar with the truncation '*', and as singular and plural terms.

$\ddagger$ The number of retrievals produced by the respective CPG terms per search engine in the preliminary study.

$\S$ Defined by Google Scholar as "Results I-10 of about...."

" The number of guidelines identified in SUMSearch by the combination of a CPG term and the MeSH term "back pain".

$*=$ truncation $;$ CPG = Clinical Practice Guideline; GLAD = GuideLine And Disease.

reviewed. Retrievals were declared "relevant" if the detected CPG fulfilled specific criteria with regard to content, language, and publication date (Figure 1). One reviewer classified the retrievals according to these criteria; these results were perused by three additional reviewers. The retrievals from Google Scholar and SUMSearch were then finally classified into two categories: either "unique and relevant" or "non-relevant or duplicate".

We recorded the number of identified CPGs per GLAD strategy (for all diseases) and per search engine, and documented the corresponding intersections of CPGs (Figure 2).

\section{Diagnostic test parameters}

The sensitivity, specificity, and NNR of the three GLAD strategies were calculated per search strategy in each search engine following the terminology of diagnostic tests, using the defined gold standard as a reference (Table 3) [26]. It should be noted that these diagnostic parameters will change depending on the reference pool.

Sensitivity for a given strategy is the proportion of identified unique and relevant guidelines among the total number of unique and relevant retrievals identified by the reference search; specificity is the proportion of non-relevant retrievals and duplicates not retrieved by this strategy among the total number of non-relevant retrievals and duplicates not retrieved by the reference search; precision is the proportion of retrieved guidelines that are unique and relevant among the total number of retrievals. The NNR (1/precision) is defined as the number of non-relevant and duplicate retrievals that have to be screened to find one relevant retrieval [33]. For all parameters, 95\% confidence intervals were calculated based on simple normal approximation for proportions.

\section{Results}

The application of the three GLAD search strategies per search engine for each of the nine diseases (totalling 27 searches per engine) yielded a total of 2830 retrievals (for the nine pooled diseases); 987 (34.9\%) in Google Scholar and $1843(65.1 \%)$ in SUMSearch. The manual review identified a total of 119 unique and relevant CPGs (reference standard) in both search engines (Table 4). SUMSearch detected 105, and Google Scholar detected 48 relevant guidelines, of which 71 and 14 were unique and relevant, respectively (Figure 2 ).

Table 5 shows the retrieval performances of the three GLAD strategies in SUMSearch and Google Scholar. In SUMSearch, the search strategy including the term "guideline" yielded the highest sensitivity $(81.5 \%)$, the lowest specificity $(74.3 \%)$, and an NNR of 8.2 (meaning that about 8 retrievals had to be reviewed to find one unique and relevant guideline). The search strategy including the term "practice guideline" yielded the highest specificity $(89.5 \%)$, the lowest sensitivity $(40.3 \%)$, and the lowest 
Table 2: Allocation of retrievals in the manual review (3 GLAD-strategies, 9 diseases)

\begin{tabular}{|c|c|c|c|}
\hline Allocation of retrievals & Unique and relevant retrievals & $\begin{array}{c}\text { Non-relevant retrievals and } \\
\text { duplicates }\end{array}$ & Total \\
\hline \multicolumn{4}{|l|}{ SUMSearch } \\
\hline Strategy tested & $\begin{array}{l}\text { unique and relevant retrievals; no } \\
\text { duplicates }\end{array}$ & $\begin{array}{c}\text { non-relevant retrievals removed } \\
\text { from manual review; duplicates of: } \\
\text { - relevant retrievals } \\
\text { - non-relevant retrievals }\end{array}$ & $\begin{array}{c}\text { raw retrievals; duplicates between } \\
\text { PubMed and NGC }\end{array}$ \\
\hline Strategies not tested & $\begin{array}{l}\text { unique and relevant retrievals; no } \\
\text { duplicates }\end{array}$ & $\begin{array}{l}\text { non-relevant retrievals removed } \\
\text { from manual review; duplicates of } \\
\text { and between: } \\
\text { - relevant retrievals } \\
\text { - non-relevant retrievals }\end{array}$ & $\begin{array}{c}\text { raw retrievals; duplicates of and } \\
\text { between retrievals }\end{array}$ \\
\hline All strategies & $\begin{array}{c}\text { unique and relevant retrievals; no } \\
\text { duplicates }\end{array}$ & $\begin{array}{l}\text { non-relevant retrievals removed } \\
\text { from manual review; duplicates of } \\
\text { and between: } \\
\text { - relevant retrievals } \\
\text { - non-relevant retrievals }\end{array}$ & $\begin{array}{c}\text { raw retrievals; duplicates of and } \\
\text { between retrievals }\end{array}$ \\
\hline
\end{tabular}

\section{Google Scholar}

\section{Strategy tested}

Strategies not tested

All strategies unique and relevant retrievals; no
duplicates

unique and relevant retrievals; no duplicates

unique and relevant retrievals; no duplicates non-relevant retrievals removed from manual review; duplicates of: - relevant retrievals

- non-relevant retrievals non-relevant retrievals removed from manual review; duplicates of and between: - relevant retrievals

- non-relevant retrievals non-relevant retrievals removed from manual review; duplicates of and between:

- relevant retrievals

- non-relevant retrievals raw retrievals; duplicates between singular and plural

raw retrievals; duplicates of and between retrievals

raw retrievals; duplicates of and between retrievals
NNR (7.0). The search strategy including the term "recommendation" did not achieve the best result for any performance parameter - neither primary nor secondary and yielded a sensitivity of $60.5 \%$, a specificity of $76.3 \%$, and a NNR of 9.9.

In Google Scholar, the search strategy including the term "guideline" yielded the highest sensitivity (31.9\%), the lowest specificity (78.1\%) and a NNR of 16.7. The search strategy including the term "practice guideline" yielded the highest specificity (95.7\%), a sensitivity of $11.8 \%$, and the lowest NNR (9.3). The search strategy including the term "recommendation" yielded the lowest sensitivity $(8.4 \%)$, a specificity of $92.1 \%$, and the highest NNR (22.4). In the two latter strategies, the low sensitivity seems to be a trade-off for the high specificity.

In summary, the GLAD search strategies showed a better retrieval performance in SUMSearch than in Google Scholar. The performance pattern between search engines was similar: in both search engines, the best results for the primary performance parameters were achieved by strate-

Table 3: Formula for calculating retrieval performance parameters for search strategies*

\begin{tabular}{lcc}
\hline & Manual review \\
\hline $\begin{array}{l}\text { Search terms } \\
\text { Detected }\end{array}$ & Meets criteria (unique, relevant CPGs) & Does not meet criteria (non-relevant CPGs or duplicates) \\
Not detected & a & b \\
\hline
\end{tabular}

*Following the methodology of the Hedges group (see text): sensitivity $=a /(a+c)$; specificity $=d /(b+d)$; precision $=a /(a+b)$; total sample $(a l l$ unscreened reviewed retrievals) $=\mathrm{a}+\mathrm{b}+\mathrm{c}+\mathrm{d} ; \mathrm{NNR}=1 /$ precision.

$\mathrm{CPG}=$ clinical practice guideline. 


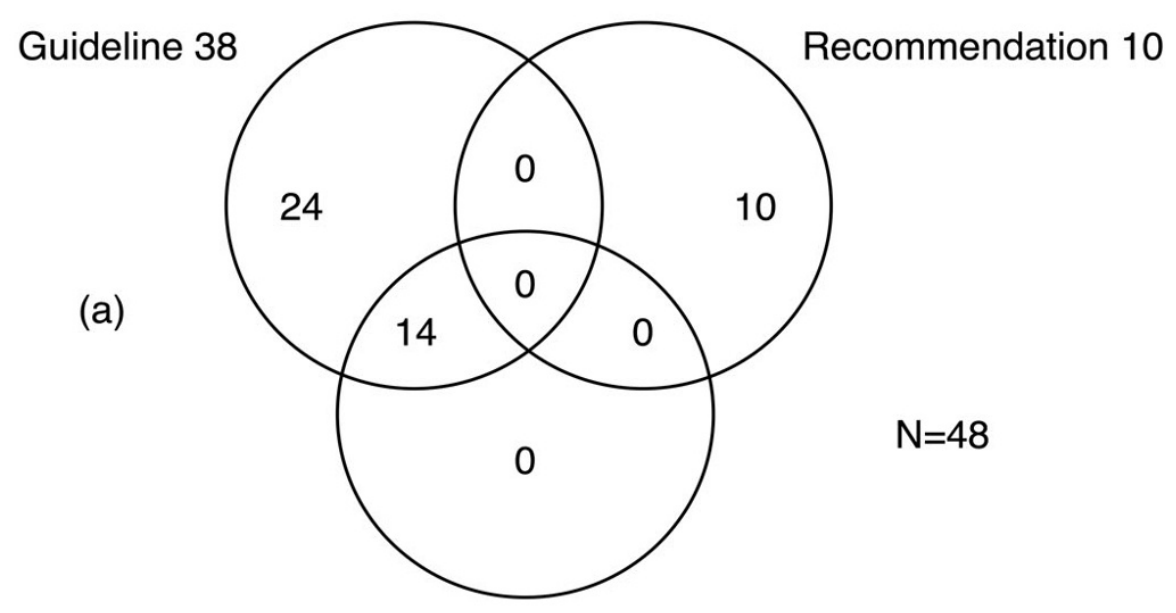

Practice Guideline 14

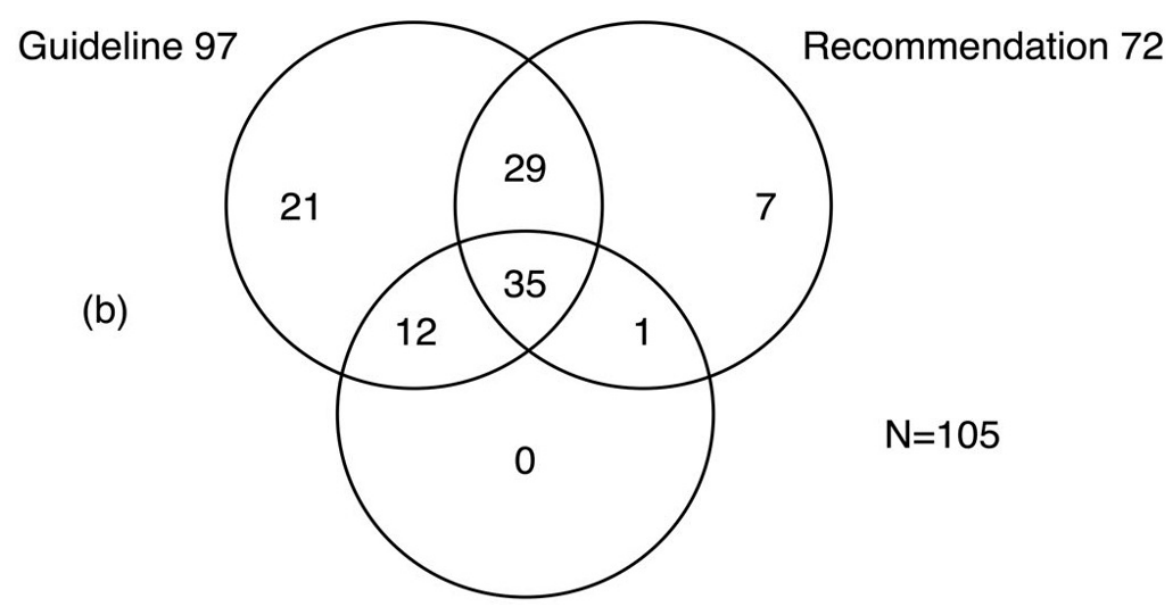

Practice Guideline 48

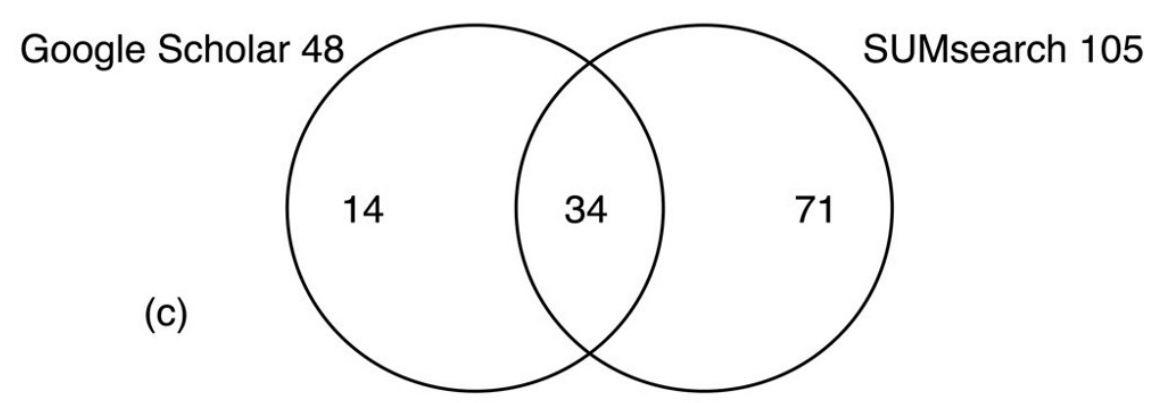

$\mathrm{N}=119$

\section{Figure 2}

Intersections of unique and relevant clinical practice guidelines of nine tested diseases. (a) Per CPG term in Google Scholar (b) Per CPG term in SUMSearch (c) All CPG terms combined (SUMSearch and Google Scholar). CPG = clinical practice guideline. 
Table 4: Retrievals obtained by application of GLAD search strategies in SUMSearch and Google Scholar

\begin{tabular}{|c|c|c|c|}
\hline Strategy Search terms & Meets criteria & Does not meet criteria & Total \\
\hline SUMSearch & & & $1843^{\dagger}$ \\
\hline \multicolumn{4}{|l|}{ Guideline* } \\
\hline Detected & 97 & 697 & 794 \\
\hline Not detected & 22 & 2014 & 2036 \\
\hline \multicolumn{4}{|l|}{ Recommendation* } \\
\hline Detected & 72 & 643 & 715 \\
\hline Not detected & 47 & 2068 & 2115 \\
\hline \multicolumn{4}{|l|}{ Practice guideline* } \\
\hline Detected & 48 & 286 & 334 \\
\hline Not detected & 71 & 2425 & 2496 \\
\hline Google Scholar & & & $987 \dagger$ \\
\hline \multicolumn{4}{|l|}{ Guideline/s } \\
\hline Detected & 38 & 595 & 633 \\
\hline Not detected & 81 & 2116 & 2197 \\
\hline \multicolumn{4}{|l|}{ Recommendation/s } \\
\hline Detected & 10 & 214 & 224 \\
\hline Not detected & 109 & 2497 & 2606 \\
\hline \multicolumn{4}{|l|}{ Practice guideline/s } \\
\hline Detected & 14 & 116 & 130 \\
\hline Not detected & 105 & 2595 & 2700 \\
\hline Total & 119 & 2711 & 2830 \\
\hline
\end{tabular}

† Number of pooled retrievals for the nine MeSH terms in each search engine.

$*=$ truncation; GLAD = Guideline And Disease.

gies including the term "guideline" (highest sensitivity) and "practice guideline" (lowest NNR). This means that the former strategy had the most comprehensive detection of unique and relevant CPGs, i.e. it detected the highest number of unique and relevant retrievals among the reference retrievals. The latter required the lowest time investment in a search, i.e. it required the lowest number of retrievals to identify a relevant retrieval. None of the tested strategies combined the highest sensitivity and lowest NNR. The best results for the secondary performance parameter (highest specificity) were also achieved by the strategy including the term "practice guideline", meaning that this strategy disregarded the highest proportion of non-relevant retrievals.

With regard to the NNR, about $30 \%$ more time was required in Google Scholar than in SUMSearch to detect a relevant CPG (using the strategy including "practice guideline") (Table 5). Concerning the primary performance parameter "sensitivity" and the search term "guide-

Table 5: Retrieval performance of search strategies in SUMSearch and Google Scholart

\begin{tabular}{lccc}
\hline Search strategy & Sensitivity (\%) & Specificity (\%) & NNR \\
\hline SUMSearch & & & \\
Guideline* & $81.51(74.53$ to 88.49$)$ & $74.29(72.64$ to 75.94$)$ & $8.18(6.90$ to 10.05$)$ \\
Recommendation* & $60.50(51.72$ to 69.28$)$ & $76.28(74.67$ to 77.89$)$ & $9.93(8.14$ to I2.72) \\
Practice guideline* & $40.34(31.52$ to 49.16$)$ & $89.45(88.29$ to 90.61$)$ & $6.96(5.52$ to 9.43$)$ \\
Google Scholar & & & $16.67(12.76$ to 24.04$)$ \\
Guideline/s & $31.93(23.56$ to 40.30$)$ & $78.05(76.50$ to 79.60$)$ & $22.42(13.97$ to 56.82$)$ \\
Recommendation/s & $8.40(3.42$ to I3.38) & $92.11(91.09$ to 93.13$)$ & $9.29(6.21$ to I8.38) \\
Practice guideline/s & $11.76(5.98$ to 17.54$)$ & $95.72(94.96$ to 96.48$)$ & \\
\hline
\end{tabular}

† $95 \%$ confidence intervals in brackets. $\ddagger N N R=$ number needed to read.

$*=$ truncation. 
line", Google Scholar only detected about $40 \%$ of the relevant CPGs that SUMSearch identified (Tables 4 and $5)$.

Google Scholar showed better results only for the secondary performance parameter "specificity" (identification of about $7 \%$ more non-relevant retrievals with the search strategy including the term "practice guideline"; Tables 4 and 5).

\section{Discussion}

The aim of this study was to provide clinicians with useful search strategies to efficiently identify relevant CPGs in SUMSearch and Google Scholar. We therefore compared the retrieval performance of GLAD (GuideLine And Disease) strategies in SUMSearch and Google Scholar, using a manual review of retrievals as a reference standard. As the reference standard was solely defined by means of the manual search of the raw retrievals in SUMSearch and Google Scholar and only includes CPGs identified by these engines, it cannot be universally transferred to searches in other search engines or bibliographic databases. It should also be noted that since conducting our search, changes have been made to both SUMSearch and Google Scholar. Therefore, the replicability of results may be affected. A further limitation of this study may be due to the fact that the reviewers were not blinded as to which search engine produced the CPG. They were not blinded because, as stated, the methodological procedures were not specified a priori and were conducted as an iterative process. However, as none of the reviewers previously routinely used either SUMSearch or Google Scholar, we believe it is unlikely that bias caused by a preference for a specific search engine was a relevant factor.

\section{Search methods in SUMSearch and Google Scholar}

The GLAD strategies showed a better retrieval performance when applied in SUMSearch than in Google Scholar. However, one needs to consider that our approach was based on the specifications developed for searches in SUMSearch; therefore, the transfer of a "SUMSearch-specific" strategy to Google Scholar might have been unfavourable for its retrieval performance. To our knowledge, there is no 'best search strategy' for CPGs - or one that is better than our GLAD strategy - available for Google Scholar.

The strength of SUMSearch lies in its expertise in the medical sciences. Since its launch, the search strategies of users have been continuously reviewed. This process has led to the current recommendations for search strategies. SUMSearch's structural characteristic as a meta-search engine in scientific sources supports the specificity of its retrieval performance. The handling of retrievals in SUMSearch is convenient. The links presented in the search results are in reference format and lead directly to the article or abstract of the retrieval.

It remains open as to whether the ongoing changes, such as the inclusion of additional filters, in the structure of Google Scholar (which is still in its beta version) will lead to an improvement in retrieval performance. Due to its intuitive approach, Google Scholar has been described as especially quick in locating frequently cited articles and the proverbial "needle in a haystack" [18]. Despite this commendation, search strategies for CPGs in Google Scholar need to be improved and defined more specifically. The handling of retrievals can be quite laborious: Web links refer to on- and offline sources, as well as to open-access sources and sources where registration is required (e.g. publishers' websites) [22], and sometimes lead to "dead links". Abstracts are not always accessible and open-access items are not specially marked. Furthermore, Google Scholar's coverage is incomplete; less than $10 \%$ of PubMed records are searched, and it only partially covers publishers' and societies' archives - again, the character of the archives is not disclosed in detail $[19,20,22,23]$. Changes in PubMed, which plans to make its web page titles more descriptive [34], may improve Google Scholar's performance in this database. Google Scholar also searches the contents of databases with a time-lag. It has been noted in online panels that the timelag for indexing PubMed has been reduced from a year to about five months [35,36].

\section{Retrieval performance results}

In SUMSearch as well as in Google Scholar, we found that the term "practice guideline" is the CPG-term that should be included in a GLAD strategy by the clinician under time pressure who is looking for quick answers to a clinical problem by means of a CPG. Although this strategy may not identify all relevant CPGs, it has a high probability of detecting a sufficient number to answer the question posed. This is because it has a low NNR and a high specificity, and is therefore likely to detect relevant retrievals quickly without having to review an excessive number of non-relevant ones. If the clinician wants to gain a comprehensive overview of CPGs on a certain topic, a GLAD search strategy including the term "guideline" should be used; this was the term with the highest sensitivity. Although this strategy is more time consuming, it considerably increases the chances of detecting a large number of relevant CPGs. However, the dilemma in which the searcher finds him- or herself, time pressure on the one hand and the desire to detect as many relevant CPGs as possible on the other hand, cannot be solved to complete satisfaction. 


\section{Conclusion}

Google Scholar is not a useful tool to search efficiently for CPGs. The advantage of being intuitive does not make up for the laborious and time consuming handling of the retrievals when screening for CPGs. We recommend SUMSearch as a starting point for a search to gain an overview of available CPGs. It specialises in the medical field and has the advantages of a meta-search engine that searches medical databases simultaneously. The retrievals are accessed quickly and reliably by links, enabling swift screening. However, neither SUMSearch nor Google Scholar can replace the commonly used CPG searches in portals/websites of organisations that publish CPGs and bibliographical databases.

The developed GLAD strategy is easy to use and to remember. It is applicable in both SUMSearch and Google Scholar. In SUMSearch, however, the GLAD strategy shows a superior retrieval performance.

We did not identify the "ideal" CPG term for a GLAD strategy. The term "guideline" should be used if the aim is to detect a comprehensive pool of CPGs, and "practice guideline" should be used if the aim is to rapidly identify CPGs.

In future, the introduction of a standardised index term for CPGs, analogous to MeSH terms, may facilitate identification by search engines and help to improve retrieval performance. A further vision for the combination of the advantages of both search principles - the federated search of Google Scholar and the meta-search of SUMSearch - could lead to the development of a 'Medical Internet Portal'. In combination with a standardised CPGindex term, relevant CPGs could be identified more quickly and comprehensively.

\section{List of abbreviations}

CPG: Clinical Practice Guideline

DARE: Database of Abstracts of Reviews of Effects

GLAD: GuideLine And Disease

MeSH: Medical Subject Heading

NGC: National Guideline Clearinghouse

NNR: Number Needed to Read

\section{Competing interests}

The author(s) declare that they have no competing interests.

\section{Authors' contributions}

This study was initiated by HK and MF. All authors provided intellectual content to the literature search and the data analysis. The literature search was conducted by $\mathrm{AH}$. Data analysis was conducted by AH and GS. The draft of the manuscript was prepared by AH. All authors commented on and approved the final manuscript.

\section{Acknowledgements}

The authors thank Natalie McGauran for editorial support.

All authors and acknowledged persons are employees of the Institute for Quality and Efficiency in Health Care. No additional funding was received.

\section{References}

I. Straus SE, Richardson WS, Glasziou P, Haynes RB: Introduction. In Evidence-Based Medicine: How to Practice and Teach EBM 3rd edition. Edinburgh , Elsevier; 2005: I-12.

2. Committee to Advise the Public Health on Clinical Guidelines, Institute of Medicine: Definitions of Key Terms. In Clinical Practice Guidelines: Directions for a New Program Edited by: Field MJ, Lohr KN. Washington, D.C. , National Academy Press; 1990:33-5I.

3. Woolf SH, Grol R, Hutchinson A, Eccles M, Grimshaw J: Clinical guidelines: Potential benefits, limitations, and harms of clinical guidelines. BMJ 1999, 3 | 8(7 | 82):527-530.

4. Ely JW, Osheroff JA, Ebell MH, Chambliss ML, Vinson DC, Stevermer J], Pifer EA: Obstacles to answering doctors' questions about patient care with evidence: qualitative study. BMJ 2002, 324(7339):710.

5. Feder G, Eccles M, Grol R, Griffiths C, Grimshaw J: Clinical guidelines: Using clinical guidelines. BM] 1999, 3 18(7185):728-730.

6. National Guideline Clearinghouse [http://www.guideline.gov/]

7. National Library for Health: Clinical Knowledge Summaries [http://cks.library.nhs.uk/clinical knowledge]

8. CMA Infobase: clinical practice guidelines [http://mdm.ca/cpg snew/cpgs/index.asp]

9. SUMSearch [http://sumsearch.uthscsa.edu]

10. Badgett R, Paukert J, Levy L: The Evolution of SUMSearchTM for Teaching Clinical Informatics to Third-year Medical Students. Acad Med 200I, 76(5):54I.

II. Badgett R: SUMsearch: evidence-based Internet searching. He@lth Information on the Internet 2000, 14:6-7.

12. Haynes RB, Wilczynski NL: Optimal search strategies for retrieving scientifically strong studies of diagnosis from Medline: analytical survey. BMJ 2004, 328(7447): 1040-1044.

13. Badgett R: SUMsearch - More Details. [http://sumsearch.uth scsa.edu/MoreDetail.htm].

14. Badgett R: SUMsearch - Details. [http://sumsearch.uthscsa.edu/ details.htm].

15. Google Scholar [http://scholar.google.com]

16. Sadeh T: Google Scholar Versus Metasearch Systems. High Energy Physics Libraries Webzine 2006.

17. About Google Scholar [http://scholar.google.com/scholar/
[ about.html]

18. Giustini D: How Google is changing medicine. BMJ 2005, 33 I (753I): | 1487-I 488.

19. Giustini D, Barsky E: A look at Google Scholar, PubMed, and Scirus: comparisons and recommendations. Journal of the Canadian Health Libraries Association 2005, 26(3):85-89.

20. Jacsó P: Google Scholar Beta. In Péter's Digital Reference Shelf Thomson Gale; 2004.

21. Payne D: Google Scholar Welcomed. The Scientist 2004, 5:2004||23-0I.

22. Vine R: Google Scholar. J Med Libr Assoc 2006, 94(I):97-99.

23. Henderson J: Google Scholar: A source for clinicians? CMA 2005, I 72(I 2): I549-1550.

24. Haynes RB, Kastner M, Wilczynski N, The Hedges Team: Developing optimal search strategies for detecting clinically sound and relevant causation studies in EMBASE. BMC Medical Informatics and Decision Making 2005, 5(I):8.

25. Haynes RB, McKibbon KA, Wilczynski NL, Walter SD, Werre SR, The Hedges Team: Optimal search strategies for retrieving sci- 
entifically strong studies of treatment from Medline: analytical survey. $B M J$ 2005, 330(750 I): I I79-I I 84.

26. Haynes RB, Wilczynski NL, McKibbon KA, Walker CJ, Sinclair JC: Developing optimal search strategies for detecting clinically sound studies in MEDLINE. J Am Med Inform Assoc 1994, I(6):447-458.

27. Holland J, Wilczynski NL, Haynes RB, The Hedges Team: Optima search strategies for identifying sound clinical prediction studies in EMBASE. BMC Medical Informatics and Decision Making 2005, 5(I): II.

28. Wilczynski NL, Haynes RB, The Hedges Team: Developing optimal search strategies for detecting clinically sound prognostic studies in MEDLINE: an analytic survey. BMC Medicine 2004, 2(I):23

29. Wilczynski NL, Haynes RB, The Hedges Team: EMBASE search strategies for identifying methodologically sound diagnostic studies for use by clinicians and researchers. BMC Medicine 2005, 3(I):7.

30. Wilczynski NL, Morgan D, Haynes RB, The Hedges Team: An overview of the design and methods for retrieving high-quality studies for clinical care. BMC Medical Informatics and Decision Making 2005, 5(I):20.

31. Wong SS, Wilczynski NL, Haynes RB: Developing optimal search strategies for detecting clinically sound treatment studies in EMBASE. J Med Libr Assoc 2006, 94(I):4 I-47.

32. Lelgemann M, Cox M, Thalau F, Thole H, Thomeczek C, Trapp H, Ollenschläger G: Durchführung eines Leitlinien-Clearingverfahrens. In Das Deutsche Leitlinien-Clearingverfahren 1999-2005. Hintergrund, Zielsetzung, Ergebnisse - Abschlussbericht Edited by: Ärztliches Zentrum für Qualität in der Medizin . Norderstedt, Books on Demand; 2006:20-26.

33. Bachmann LM, Coray R, Estermann P, ter Riet G: Identifying Diagnostic Studies in MEDLINE: Reducing the Number Needed to Read. J Am Med Inform Assoc 2002, 9(6):653-658.

34. Nahin AM: NCBI to Introduce Changes to the Entrez System - Beta Version Available for Preview. NLM Technical Bulletin 2007, 355(Mar-Apr):e7.

35. Vine R: Google Scholar is a full year late indexing PubMed content. In Sitelines - Ideas about Web Searching Search Portfolio; 2005.

36. Vine R: Google Scholar gets better at indexing PubMed content, but it's still several months behind. In Sitelines - Ideas about Web Searching Search Portfolio; 2006.

\section{Pre-publication history}

The pre-publication history for this paper can be accessed here:

http://www.biomedcentral.com/1471-2288/7/28/prepub
Publish with Bio Med Central and every scientist can read your work free of charge

"BioMed Central will be the most significant development for disseminating the results of biomedical research in our lifetime. "

Sir Paul Nurse, Cancer Research UK

Your research papers will be:

- available free of charge to the entire biomedical community

- peer reviewed and published immediately upon acceptance

- cited in PubMed and archived on PubMed Central

- yours - you keep the copyright

Submit your manuscript here:

http://www.biomedcentral.com/info/publishing_adv.asp
BioMedcentral 